\title{
Positrons and Electrons at HERA and HERMES
}

Caroline Riedl, and HERMES collaboration

Citation: AIP Conference Proceedings 1160, 36 (2009); doi: 10.1063/1.3232030

View online: https://doi.org/10.1063/1.3232030

View Table of Contents: http://aip.scitation.org/toc/apc/1160/1

Published by the American Institute of Physics

\section{Conference Proceedings}

Get $30 \%$ off all

Enter Promotion Code PDF30 a print proceedings! 


\title{
Positrons and Electrons at HERA and HERMES
}

\author{
Caroline Riedl \\ (on behalf of the HERMES collaboration) \\ DESY, 15738 Zeuthen, Germany
}

\begin{abstract}
The HERA electron-proton storage ring at DESY, Hamburg, provided a unique laboratory for the collection of data in deep-inelastic charged lepton-proton scattering at a center-of-mass energy of about $7 \mathrm{GeV}$ for the fixed-target experiment HERMES and at $318 \mathrm{GeV}$ for the collider experiments ZEUS and H1. HERA could be operated with both electrons and positrons. The ability of the lepton beam to polarize itself was exploited.

Data taken with the HERMES spectrometer on unpolarized and transversely polarized gaseous targets are presented. Two examples involving interference processes are chosen that are sensitive to the beam charge: the measurement of azimuthal asymmtries in deeply-virtual COMPTON scattering and the search for a two-photon exchange signal at HERMES.
\end{abstract}

Keywords: Deeply Virtual Compton Scattering, two-photon exchange

PACS: $13.60 .-\mathrm{r} 24.85 .+\mathrm{p}$ 13.60.Fz 14.60.Cd

\section{PHYSICS WITH TWO DIFFERENT BEAM CHARGES}

A cross-section is usually proportional to a squared amplitude. Any information on the sign of the lepton beam charge in an electromagnetic interaction is lost in this case. However, interference processes involving amplitudes with an odd net number of couplings $\propto e_{\ell}$ of the lepton beam are sensitive to the beam charge $e_{\ell}$. Two examples are presented below: the interference of Deeply-Virtual COMPTON Scattering (DVCS) with the BETHE-HEITLER (BH) process, and a transverse single-spin asymmetry arising from the interference of one- and two-photon exchange amplitudes.

On the electroweak sector, the sign of the beam charge can generate a sensitivity to the quark flavor because the gauge bosons $W^{ \pm}$carry an electric charge themselves.

\section{HERA}

HERA at DESY in Hamburg, Germany, is a storage ring for charged leptons (electrons or positrons) and protons (Fig.1). Its operation ended mid-2007 after 15 years of running. The beams were cycled through several stages of preacceleration. At an energy of 12 $\mathrm{GeV}$ and typical currents of about $40 \mathrm{~mA}$, the leptons were injected from the PETRA storage ring into HERA, where the beam was ramped to the final energy of about 27.6 $\mathrm{GeV}$ and brought to collision with the $920 \mathrm{GeV}$ proton beam in the north $(\mathrm{H} 1)$ and in the south (ZEUS) areas. In the east area (HERMES), the lepton beam passed through a target of pure hydrogen or heavier gas internal to the beam line. One fill was typically stored for about ten hours. During the last hour of each fill, HERMES was allowed to increase the target density by a factor of 10 or more, allowing the use of high density targets. The 

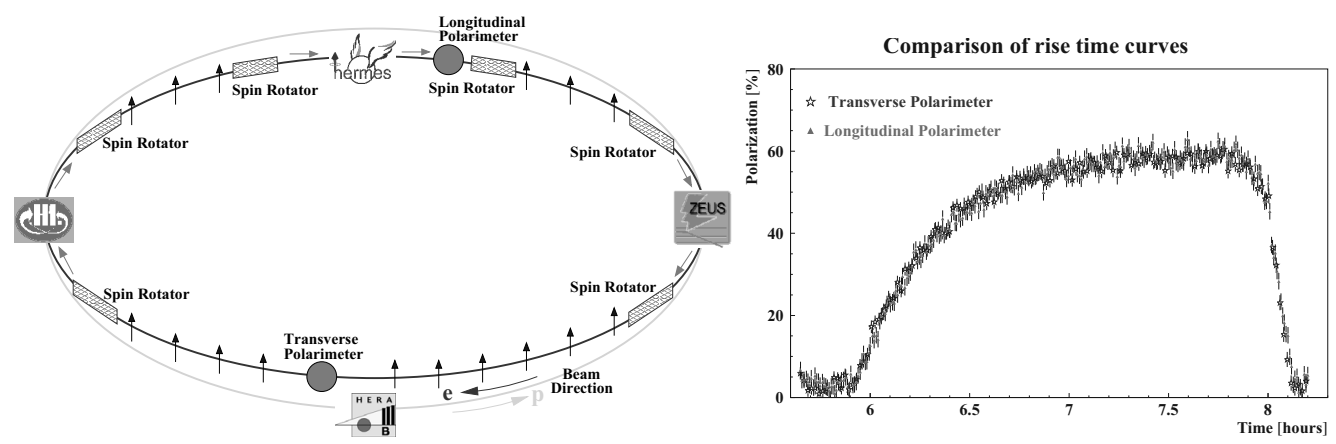

FIGURE 1. The HERA electron-proton storage ring and a rise-time curve of lepton polarization.

HERA electron machine usually contained 180 bunches (max. 220) separated by $96 \mathrm{~ns}$ and each with a length of $27 \mathrm{ps}$.

The lepton beam automatically became transversely polarized through a small spinflip amplitude in the emission of synchrotron radiation (SOKOLOV-TERNOV mechanism [1]). The polarization approached an equilibrium value asymptotically, with a time constant depending on the characteristics of the electron ring, which at HERA was about 30 $\min$ (Fig.1). It was therefore not necessary to rely on a polarized source at injection. Spin rotators upstream and downstream of the experiments provided longitudinal polarization at the interaction points.

Two COMPTON polarimeters continuously measured the beam polarization with an uncertainty of $2 \%[2,3]$. Until 2000 , the average lepton beam polarization was about $55 \%$; it deteriorated to values of about $35 \%$ after the HERA luminosity upgrade, because the machine tune was optimized for collider luminosity and not polarization. HERA operated most $(70 \%)$ of the time with positrons and protons. Positrons as beam particles bring along the feature of longer beam life times, as compared to electrons, since residual gas ions are pushed away from the beam, which is a current of particles with the same charge. The lepton beam charge has also an influence on the beam polarization due to beam-beam effects: positrons and protons interact in a repulsive way with each other, thereby defocussing the beams and decreasing the magnitude of the beam polarization.

\section{HERMES: AZIMUTHAL ASYMMETRIES IN DVCS}

\section{HERMES and DVCS}

The HERMES experiment [4] recorded data from scattering the longitudinally polarized lepton beam of HERA off an internal gas target. Events with $Q^{2}>1 \mathrm{GeV}^{2}$ were selected which contained exactly the beam lepton and one photon identified as a cluster in the calorimeter without an associated track. The recoiling target proton was not detected, instead, exclusivity of the events was achieved indirectly by a missing-mass technique. With this technique, events nonetheless contribute to the yield for which the nucleon was excited to a resonant state. The main contribution to the background of 
about $3 \%$ originates from semi-inclusive $\pi^{0}$ production and is corrected for. Between 1996 and 2005, 25k DVCS events on a polarized or unpolarized hydrogen target and $15 \mathrm{k}$ DVCS events on an unpolarized deuterium target were collected.

\section{GPDs and azimuthal asymmetry amplitudes in DVCS}

DVCS, the hard exclusive leptoproduction of real photons, can be described in the framework of Generalized Parton Distributions (GPDs), which provide a unified threedimensional picture of the nucleon subsuming both ordinary parton distribution functions and form factors (see e. g. [5]). Moreover, GPDs offer a way to access the total angular momentum carried by partons in the nucleon [6].

In DVCS, the real photon is radiated from a quark inside the proton (or nucleus), whereas in BH, it is radiated from the lepton. Since these two processes are intrinsically indistinguishable, the DVCS amplitude $\mathscr{T}_{\mathrm{DVCS}}$ and the BH amplitude $\mathscr{T}_{\mathrm{BH}}$ interfere, and the cross-section is proportional to $\left|\mathscr{T}_{\mathrm{DVCS}}\right|^{2}+\left|\mathscr{T}_{\mathrm{BH}}\right|^{2}+\left(\mathscr{T}_{\mathrm{DVCS}} \mathscr{T}_{\mathrm{BH}}^{*}+\mathscr{T}_{\mathrm{DVCS}}{ }^{*} \mathscr{T}_{\mathrm{BH}}\right)$, where the interference term is included in parentheses. At HERMES kinematics, the DVCS amplitude is supressed with respect to the BH one. Note that the squared amplitudes contain an even and the interference term an odd number of couplings to the lepton beam, making the first ones charge-independent and the latter one charge-dependent.

The BORN cross-section for a beam with longitudinal (L) polarization $P_{B}$ and charge $C_{B}$ and an unpolarized (U) target can be written as:

$$
\sigma\left(\phi ; P_{B}, C_{B}\right)=\sigma_{\mathrm{UU}}(\phi) \cdot\left[1+P_{B} \mathscr{A}_{\mathrm{LU}}^{\mathrm{DVCS}}(\phi)+C_{B} P_{B} \mathscr{A}_{\mathrm{LU}}^{\mathscr{I}}(\phi)+C_{B} \mathscr{A}_{\mathrm{C}}(\phi)\right],
$$

where $\sigma_{\mathrm{UU}}(\phi)$ is the unpolarized cross-section and $\phi$ the azimuthal angle between the lepton scattering plane and the photon production plane about the virtual photon direction. The dependences on the three other kinematic variables $x_{B}$ (the BJORKEN scaling variable), $Q^{2}$ (the negative squared four-momentum mediated by the virtual photon) and $t$ (the squared four-momentum transfer to the target nucleon) have been omitted. $\mathscr{A}_{\mathrm{LU}}^{\mathscr{I}}\left[\mathscr{A}_{\mathrm{LU}}^{\mathrm{DVCS}}\right]$ is the beam-charge [in]dependent beam-helicity asymmetry (BSA) and $\mathscr{A}_{\mathrm{C}}$ the beam-charge asymmetry (BCA). These azimuthal asymmetries are expanded in a harmonic series in $\phi$, whereby the BSAs contain only harmonics odd in $\phi$ and the BCA only terms even in $\phi$. In the analysis of experimental data, only effective asymmetries can be extracted that carry additional $\phi$-even dependencies in the denominator. To obtain the FOURIER coefficients of the harmonic expansion, hereafter called asymmetry amplitudes, data with two beam charges and helicities were combined and subject to a simultaneous fit [7].

The most important results on the hydrogen target [8] are displayed in Figs. 2 and 3 in bins of $-t, Q^{2}$ and $x_{B}$. The leading contributions to the BCA are the $\cos \phi$ amplitude and the constant term $(\cos 0 \phi)$. They both show an increasing magnitude with increasing $-t$, but have opposite signs, as theoretically expected. Both are related to the real part of the GPD $H$. The leading contribution to the charge-dependent BSA is the $\sin \phi$ moment, which is related to the imaginary part of the GPD $H$. It is found to be overall large, but it exhibits no significant kinematic dependence. 


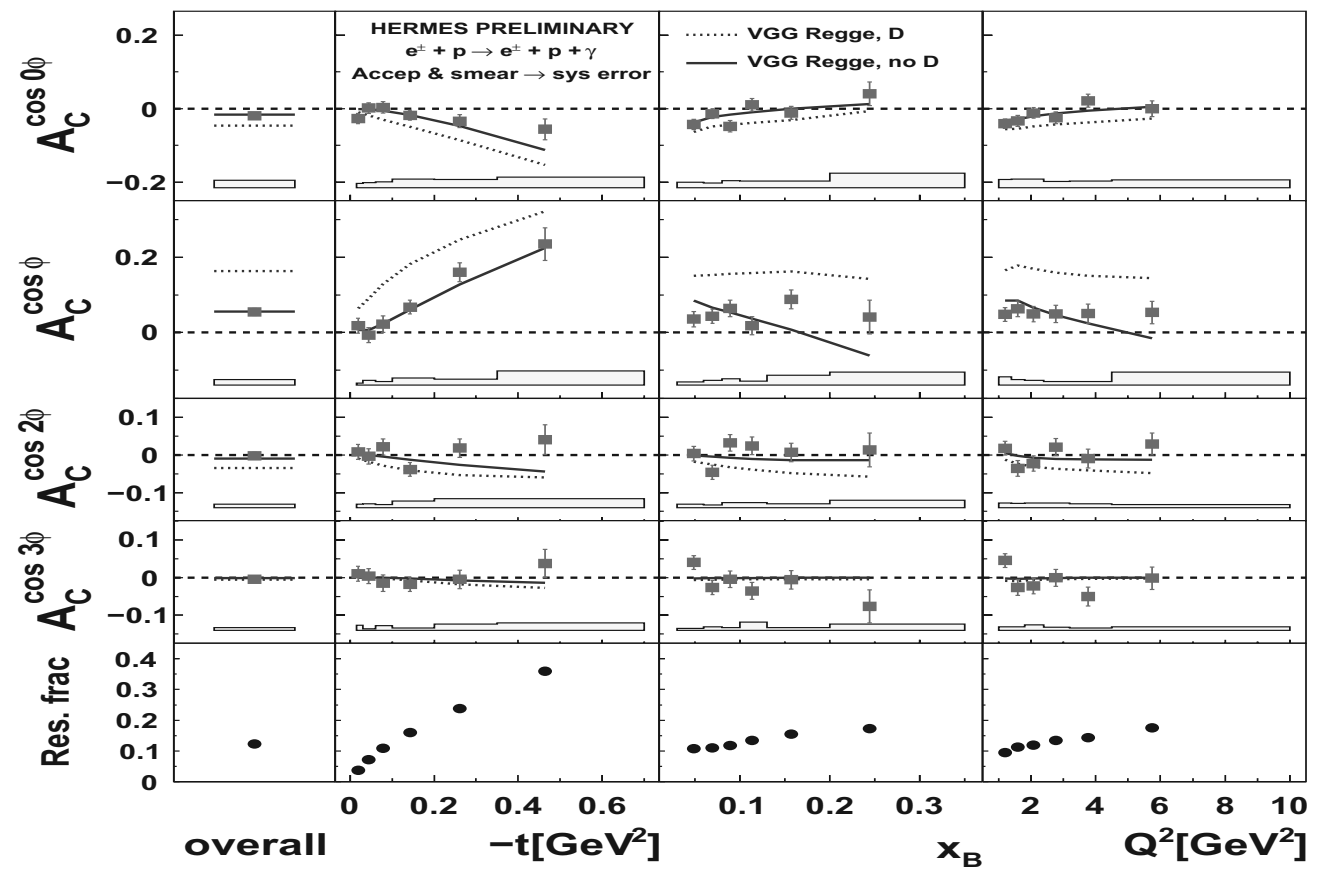

FIGURE 2. The BCA amplitudes from DVCS on the HERMES hydrogen target.

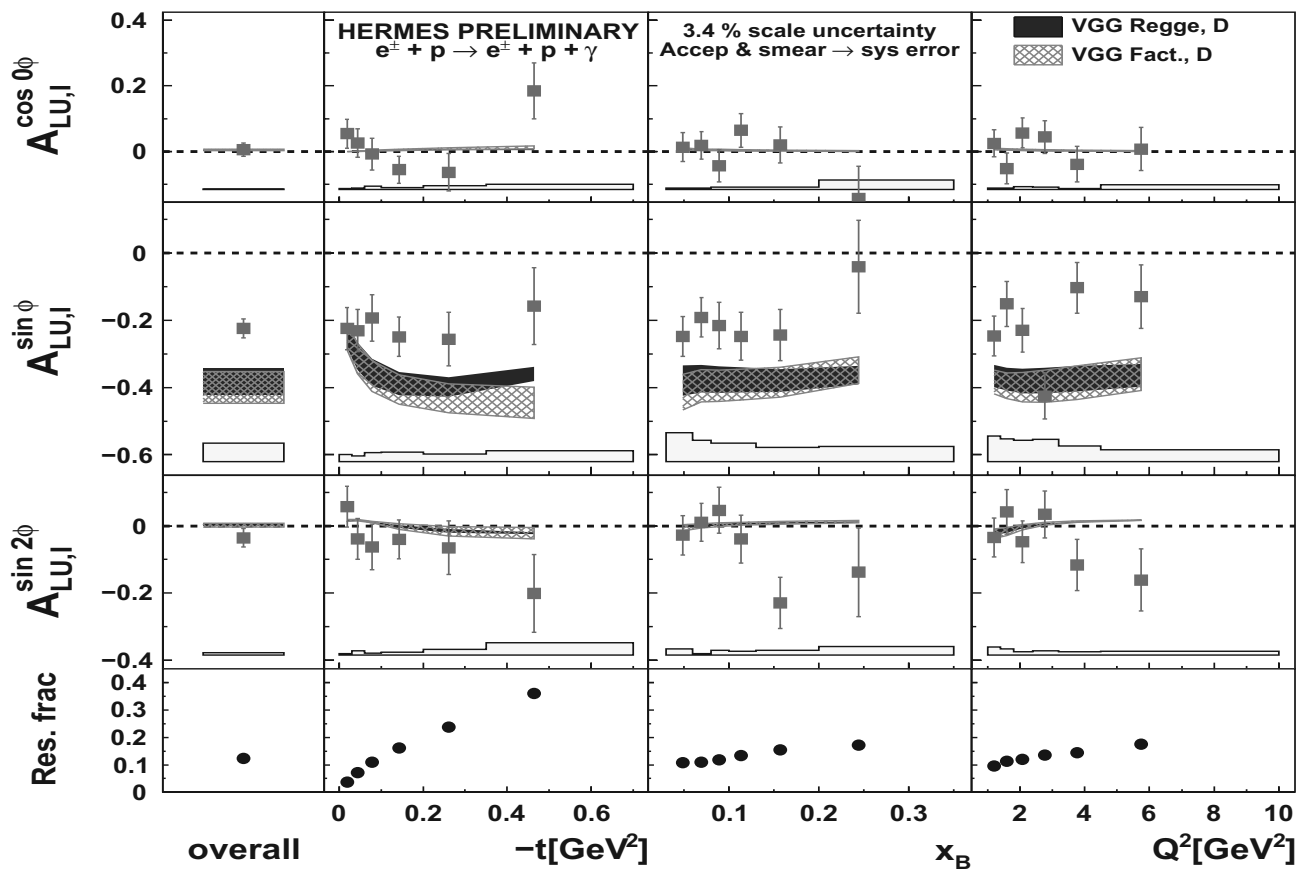

FIGURE 3. The beam-charge dependent BSA amplitudes from DVCS on the HERMES hydrogen target. 

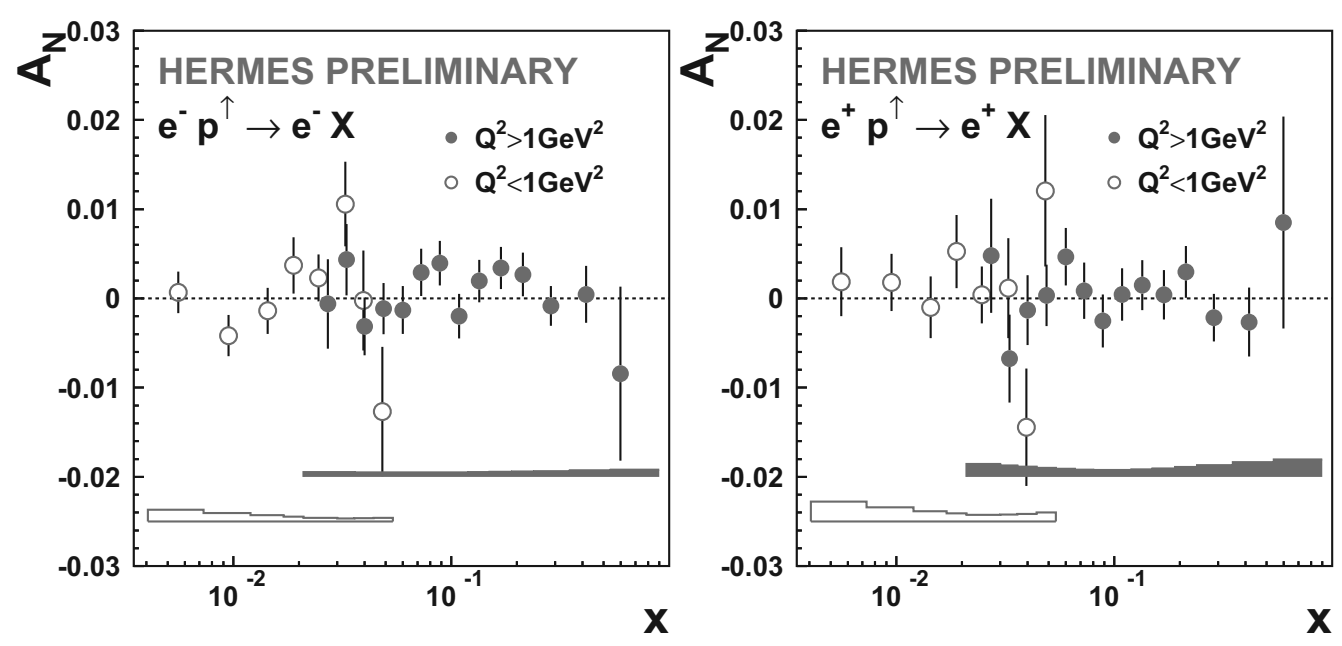

FIGURE 4. Inclusive left-right asymmetries on a transversely polarized hydrogen target measured at HERMES, left side: from an electron beam, right side: from a positron beam.

Also shown in Figs. 2 and 3 are results of two variants from a GPD model calculation $[9,10]$ based on the framework of double distributions. For the BCA, one model variant describes the data fairly well, whereas the variant including the so-called D-term is disfavored by the data. The model fails to describe the beam-charge dependent BSA. The respective lowest panels display the binwise fractions of resonant processes obtained from a Monte-Carlo simulation, which rise with increasing $-t$ up to a value of about $40 \%$. Not displayed are the amplitudes of the charge-independent BSA. They are found to be consistent with zero, as predicted by the model calculations mentioned above.

The DVCS BCA and BSA amplitudes have also been extracted from HERMES data on an unpolarized deuterium target [11]. The leading amplitudes are found to be compatible with the hydrogen results in most kinematic bins.

Of interest is also the transverse target polarization asymmetry in DVCS. Between 2002 and 2005, data have been taken at HERMES on a transversely polarized hydrogen target [7]. The azimuthal amplitudes of this transverse asymmetry relate to the imaginary part of a combination of the GPDs $H$ and $E$. The measurement helps to further constrain GPD models and to determine a model-dependent constraint on a linear combination of the total angular momentum carried by $\mathrm{u}$ - and d-quarks.

In the last two years of HERA operation, a much higher statistics data set from high-luminosity running on unpolarized targets was recorded by HERMES, including information from a recoil particle detector. This allows one to detect the intact target proton in exclusive reactions and to thus require true exclusivity. Using the additional information, it will be possible to separate the elastic BH and the DVCS process from their corresponding resonant processes. 


\section{HERMES: SEARCH FOR A TWO-PHOTON EXCHANGE SIGNAL}

To date, evidence for non-zero two-photon exchange exists only from elastic electronproton scattering. Two-photon exchange amplitudes serve as a possible explanation for the disprepancy in the elastic form factor measurements of the proton using the ROSENBLUTH separation method and the recoil polarization transfer method [12].

In inelastic scattering, no clear signature of two-photon exchange effects has yet been obtained. A transverse single-spin asymmetry in inclusive deep-inelastic scattering is forbidden in one-photon exchange approximation, but it is expected to be non-zero in the presence of two-photon effects through the interference of two-photon exchange with one-photon exchange [13]. The asymmetry arises from the correlation between the polarization three-vector of the polarized particle (beam or target) and the fourmomenta of the nucleon and leptons, reaching its largest value when the spin vector is perpendicular to the lepton scattering plane. A calculation based on certain model assumptions [14] for the future JLab Hall-A E-07-013 experiment yields expectations for the asymmetry of order $10^{-4}$ at the kinematics of that experiment. Because of the odd number of couplings to the beam $(1+2)$, the asymmetry is expected to change sign for different lepton beam charges.

Inclusive HERMES data are presented that were taken on a transversely polarized hydrogen target, covering the kinematic range $0.007<x_{B}<0.900$ and $0.25 \mathrm{GeV}^{2}<$ $Q^{2}<20 \mathrm{GeV}^{2}$ and requiring the invariant squared mass of the final hadronic state to be $W^{2}>4 \mathrm{GeV}^{2}$. Altogether, eight million events were collected. A left-right asymmetry $A_{N}$ was extracted [15] by effectively subtracting the number of detected leptons in the right half of the detector $\left(N_{R}\right)$ from that detected in the left one $\left(N_{L}\right)$ :

$$
A_{\mathrm{N}}=\frac{\sqrt{\frac{N_{R}^{\uparrow}}{L_{P}^{\uparrow}} \frac{N_{L}^{\downarrow}}{L_{P}^{\downarrow}}}-\sqrt{\frac{N_{L}^{\uparrow}}{L_{P}^{\uparrow}} \frac{N_{R}^{\downarrow}}{L_{P}^{\downarrow}}}}{\sqrt{\frac{N_{R}^{\uparrow}}{L^{\uparrow}} \frac{N_{L}^{\downarrow}}{L^{\downarrow}}}+\sqrt{\frac{N_{L}^{\uparrow}}{L^{\uparrow}} \frac{N_{R}^{\downarrow}}{L^{\downarrow}}}} \simeq A_{\text {true }}\left(1+\frac{P^{\uparrow}-P^{\downarrow}}{P^{\uparrow}+P^{\downarrow}}\right),
$$

where the superscript $\uparrow(\downarrow)$ indicates "upward" ("downward") target polarization $P$, and $L_{(P)}$ is the (polarization weighted) luminosity. This expression for $A_{N}$ causes false asymmetries due to acceptance effects to cancel. Moreover, since the target polarization sign was flipped rapidly every $1-3 \mathrm{~min}, P^{\uparrow} \approx P^{\downarrow}$, and the measured asymmetry is expected to be the true one. The asymmetry was determined separately for data taken with an electron beam and with a positron beam. The results are displayed in Fig. 4 in 14 bins in $x_{B}$ for the range $Q^{2}>1 \mathrm{GeV}^{2}$ and six bins for $Q^{2}<1 \mathrm{GeV}^{2}$. Within the experimental uncertainties, both the result for electrons and for positrons is compatible with zero on the level of a few $10^{-3}$, thus no signal of two-photon exchange is observed.

\section{ACKNOWLEDGMENTS}

I gratefully acknowledge Gunar Schnell and Mathias Vogt for the fruitful discussions and Gunar for pointing me to this workshop. 


\section{REFERENCES}

1. A. A. Sokolov and I. M. Ternov, Sov. Phys. Doklady 8 (1964) 1203.

2. D. P. Barber et al., Nucl. Instr. and Meth. A329 (1993) 79.

3. M. Beckmann et al., Nucl. Instr. and Meth. A479 (2002) 334.

4. The Hermes Collaboration, K. Ackerstaff et al., Nucl. Instr. and Meth. A417 (1998) 230.

5. A. V. Belitsky, D. Mueller and A. Kirchner, Nucl. Phys. B629 (2002) 323-392.

6. X. Ji, Phys. Rev. D55 (1997) 7114.

7. The Hermes Collaboration, A. Airapetian et al., JHEP 0806 (2008) 066.

8. D. Zeiler for the Hermes Collaboration, Proceedings of the 16th International Workshop on Deep Inelastic Scattering and QCD (DIS 2008), London, UK, arXiv:0810.5007, and the HERMES Collaboration, publication in preparation.

9. M. Vanderhaeghen, P. A. M. Guichon and M. Guidal, D60 (1999) 094017.

10. K. Goeke, M. V. Polyakov and M. Vanderhaeghen, Prog. Nucl. Phys. 47 (2001) 401.

11. H. Marukyan for the Hermes Collaboration, Proceedings of the 16th International Workshop on Deep Inelastic Scattering and QCD (DIS 2008), London, UK, and the HERMES Collaboration, publication in preparation.

12. C. E. Hyde and K. de Jager, Ann. Rev. Nucl. Part. Sci. 54 (2004) 217.

13. A. Metz, M. Schlegel and K. Goeke, Phys. Lett. B643 (2006) 319.

14. A. Afanasev, M. Strikmann and C. Weiss, Phys. Rev. D77 (2008) 014028.

15. L. De Nardo for the Hermes Collaboration, Proceedings of the 18th International Symposium on Spin Physics (SPIN08), Charlottesville, VA, USA, and the Hermes Collaboration, publication in preparation. 